\title{
La docencia universitaria con miras a la comprensión de la realidad: una mirada desde el enfoque sociocrítico.'
}

\author{
José Eduardo Padilla Beltrán ${ }^{2}$ \\ Walter Aguirre Bernal ${ }^{3}$ \\ Wilmer Hernando Silva Carreño ${ }^{4}$
}

\begin{abstract}
Resumen
La formación docente, especialmente en Latinoamérica, ha experimentado un serio replanteamiento de comprensión como fenómeno significativo de la sociedad, por lo que es pertinente fundamentarla desde un enfoque sociocrítico, estableciendo cuáles pueden ser aquellos referentes teóricos que la sustenten y como medio de empoderamiento democrático de los docentes y estudiantes, ante los emergentes retos de los paradigmas o sistemas políticos y económicos que estructuran la sociedad. Este artículo de investigación analiza la importancia de discutir y examinar las prácticas, propósitos, contexto y actores presentes en el devenir docente desde un enfoque sociocrítico, como una propuesta que facilita y lleva a desentrañar y cuestionar toda forma de dominación, mediante el establecimiento personal de una conciencia crítica y constructiva de la sociedad.
\end{abstract}

Palabras clave: pedagogía sociocrítica, formación docente, intelectual transformador, estudiante, humanización.

\footnotetext{
1. Este artículo hace parte de la investigación "Formación pedagógica e investigativa del docente universitario desde la perspectiva sociocrítica de Henry Giroux", desarrollada por el Grupo PYDES, durante los años 2010 y 2011.

2. Docente Asociado de la Universidad Militar Nueva Granada, Director del Centro de Investigaciones de la Facultad de Estudios a Distancia y Director del Grupo PYDES. Licenciado en Matemáticas, Universidad Pedagógica Nacional; Especialista en Orientación Educativa, Universidad Manuela Beltrán; Magíster en Administración y Supervisión Educativa, Universidad Externado de Colombia; Magíster en Educación con énfasis en evaluación educativa, Universidad Santo Tomás; Doctor en Educación, Newport University, U.S.A. E-mail: eduardo.padilla@unimilitar.edu.co

3. Docente de la Universidad Militar Nueva Granada, Facultad de Estudios a Distancia. Magister en Ciencias de la Educación Especialista en Pedagogía y Docencia Universitaria, Administrador de Negocios con énfasis en Finanzas, Universidad de San Buenaventura; E-mail: walter.aguirre@unimilitar.edu.co

4. Docente de la Maestría en Ciencias de la Educación en la Universidad de San Buenaventura; Licenciado en Filosofía y Magíster en Ciencias de la Educación, Universidad de San Buenaventura; Doctorando en Educación (en curso), UPN. Investigador del Grupo PYDES. E-mail:wsilva@usbbog.edu.co
} 


\title{
University teaching for the purpose of understanding reality: A sociocritical approach
}

\begin{abstract}
Teacher training, especially in Latin America, has undergone a deep reconceptualisation as a society's significant phenomenon. Therefore, it is appropriate to ground teacher training on a sociocritical approach, by establishing some theoretical points of reference that support it, and as a means of democratic empowerment for teachers and students face to emerging challenges from political and economic paradigms and systems shaping society. This research paper studies the importance of discussing and examining teaching formation practices, purposes, context and actors from a sociocritical approach, as a proposal that facilitates and leads to unravel and challenge any and all forms of domination, through an individual building a critical and constructive awareness of society.
\end{abstract}

Key words: sociocritical pedagogy, teacher education, transformative intellectual, student, humanization.

\section{El docente como intelectual transformador}

Frente a una comprensión del docente como un técnico que cumple funciones de instructor y transmisor de conocimiento, como lo enuncia Freire (1985), desde la pedagogía sociocrítica, para abordar una comprensión del docente se tiene como presupuesto relevante identificar las limitaciones de sus estudiantes y favorecer sus capacidades como bases de una formación autoconsciente que los sitúa como referente de identidad social. Por esto, cambiar el paradigma pedagógico de la formación para la competitividad, para la perpetuación de sistemas de valores deshumanizadores, implica que los procesos educativos tengan como características estructurantes una formación para la participación, la transformación, la comunicación, la contextualización; valores en últimas, propios de una formación humana y humanizadora.

Desde la perspectiva sociocrítica, uno de los puntos de partida más importantes en la concepción del docente y su formación se caracteriza por tener en cuenta 
que “(...) una práctica educativa crítica y transformadora toma muy en serio las capacidades de los docentes de teorizar, contextualizar y hacer honor a las vidas diversas de los estudiantes" (Giroux, 2003, p. 90). Y es a partir de aquí desde donde se hace necesario analizar cómo el pensamiento del docente debe ser transformado en tanto que se deben analizar no los procesos dados entre docente-estudiante, sino cómo se están dando los proceso de formación de los docentes.

La pedagogía crítica al plantear el rol del docente como intelectual transformador, exige por una parte, formar un docente como agente promotor de un cambio social no sólo en los procesos de enseñanza-aprendizaje sino en la realidad escolar. Así, su finalidad debe ser la emancipación del estudiante como persona, de modo que éste se constituya en un actor político. Por otra parte, promueve desarrollar este proyecto de lucha, de emancipación, a favor del reconocimiento de la labor docente, del rol docente en la construcción de la sociedad, de la afirmación profesional del docente, lo que incluye por ejemplo, una apuesta por la justicia en el régimen salarial (Giroux, 2003).

Giroux (1997) al caracterizar la comprensión del docente como intelectual transformador, advierte que es esencial que los educadores puedan ofrecer efectiva resistencia a los sistemas de dominio, de vigilancia y control de la educación, y puedan a la vez llevar a sus estudiantes a comprenderse como sujetos de viva transformación. Por esto, frente al devenir de aparatos ideológicos de orden político y económico que buscan de forma imperativa establecer valores universales de competencia y consumismo, esta visión de la pedagogía crítica indica que el docente debe asumir un rol de luchador por una sociedad mejor, donde la democracia es recreada como referente de calidad de vida y de dignificación humana. Así, la enseñanza se configura como un proceso político donde las pretensiones del mercado cedan ante los valores universales de mayor alcance de la democracia y porque las políticas macroeconómicas favorezcan y garanticen lo público (McLaren, 2001).

De este modo, las prácticas educativas universitarias de los docentes deben impactar constructivamente a la sociedad y a la región en la cual están inmersas y, más aún, constituirse en espacios políticos de crítica y reflexión en torno a las problemáticas que envuelven a la sociedad, denunciando y estableciendo propuestas de solución frente a las desigualdades e inequidades sociales y universitarias. El colectivo universitario debe involucrarse como agente de cambio, de transformación social, de liberación humana, de esperanza pública.

La formación universitaria requiere de docentes investigadores capaces de orientar procesos de empoderamiento en sus estudiantes, con disposición a la búsqueda constante de la justicia social a favor especialmente de los más desprotegidos, capaces de comprender la educación como un todo dinámico y dialéctico respecto a la sociedad (Giroux, 2003). Pensar al docente como 
investigador es comprender que más que un reproductor y consumidor de conocimiento es un productor del mismo.

\section{Rol del estudiante desde la sociocrítica}

Puede verse cómo el estudiante se presenta como un sujeto de aprendizaje pasivo, sin ejercer un papel relevante, participativo y menos aún, crítico de los procesos educativos que a su alrededor se desarrollan. Se le educa estandarizando un perfil profesional de ejercicio y desempeño social y laboral, en el desarrollo de individualidades y bajo una conceptualización de la educación como un requisito para acceder a un bienestar socioeconómico. Al analizar al estudiante desde un enfoque sociocrítico, se integra en la educación de forma activa, docente - estudiante - escuela, como agentes de gestión y de transformación de las experiencias, incluso conceptual.

Este proceso de relación horizontal entre el docente y el estudiante denota el carácter imprescindible de interés y análisis crítico de la escuela, su estructura, sus fines, en tanto que dicha relación ya no es normativa, institucional, sino dialéctica. Esto exige al estudiante asumir un rol activo en la participación de los procesos escolares, apropiándose del desarrollo progresivo de sus conocimientos y habilidades según su experiencia de vida. La idea es que el estudiante debe "asumirse como ser social e histórico, como ser pensante, comunicante, transformador, creador, realizador de sueños, capaz de sentir rabia porque es capaz de amar", (Freire, 1997, p. 46).

Por otro lado, el aula no puede comprenderse como un simple salón de clases, un lugar unidimensional para el desarrollo de prácticas escolares normativas y disciplinarias. Es ante todo un terreno cultural donde se desarrollan intereses, prácticas y experiencias de lucha por el poder emergente; donde se construye una cultura escolar que demanda la validación de las historias, experiencias y la construcción colectiva del conocimiento por parte de los estudiantes (McLaren, 2003).

Apple (1986), plantea que no puede darse una resistencia significativa a las ideologías de imposición económica y cultural si no se promueve un pensamiento dialógico, de acción y cambio por parte de estudiantes y docentes. Esto tendría lugar en la medida en que son capaces de reinterpretar los fenómenos, discursos y mensajes sociales, provocando situaciones de transformación social.

Desde la pedagogía crítica, para establecer la relación entre el estudiante y la comunidad, se pueden mencionar las siguientes características: el estudiante adquiere memoria histórica, esto es, conciencia de resistencia y dignidad frente 
a todo acontecimiento de sufrimiento, explotación y subyugación ideológica. Él se descubre como el agente principal para el desarrollo de un mejor estilo de vida, y se convierte en un protagonista crítico y conocedor de la problemática social que le envuelve.

No se da una educación de transformación y liberación si no se piensa y concibe personalmente el deseo y el proyecto de generar un cambio social. Si no se tiene en cuenta que el contexto sociopolítico afecta y es el horizonte de las prácticas educativas, si no se constituye como referente fundamental dar sentido y solución a las problemáticas sociales que caracterizan nuestro entorno. Se trata de posibilitar la vida escolar, las prácticas escolares, la comunidad, como centros y puntos de partida de relaciones humanas vivas, dinámicas que permiten un ambiente de desafío crítico y participativo en la construcción de la sociedad (McLaren, 1997).

Posibilitar que los estudiantes se conviertan en sujetos críticos, en ciudadanos críticos, puede tener como base el cambio objetivo de las prácticas escolares del docente. No es el desarrollo de procesos verticales, sino la integración y participación directa de los estudiantes, de sus experiencias e historias en la ejecución de éstos lo que puede generar este cambio de rol en los educandos. Para constituir directrices integrales de una formación crítica, se pueden considerar, entre otras cosas: permitir la participación directa y dinámica de los estudiantes en su propio proceso de aprendizaje, dar oportunidad para que sean investigadores y gestores creativos de su formación, posibilitar el desarrollo de sus habilidades intelectuales y físicas, estimular el interés y la curiosidad por conocer y pensar el mundo y sus problemáticas. El docente debe así propiciar situaciones en las que se desarrollen estas prácticas y orientar los procesos educativos de forma significativa para la formación integral y, especialmente, humana de sus educandos (Giroux, 2006).

\section{Diseño metodológico}

La presente investigación se enmarcó dentro de un enfoque cualitativo, trabajando asimismo desde el paradigma crítico social y utilizando como estrategia metodológica para resolver el problema de investigación, la teoría fundamentada, la cual fue propuesta por Glaser y Strauss en 1960. Ésta, considerada como una forma de hacer investigación que exige un contacto directo con la realidad, su énfasis es la construcción de teorías de forma inductiva a partir de un conjunto de datos, hace una consideración más por el caso que por la variable, estudia la vida social del individuo (Clarke, 2003), y además, tiene como finalidad esencial abordar la realidad para transformarla, puesto que ésta depende de las circunstancias, es una construcción y reconstrucción intersubjetiva, donde la acción comunicativa es la base del conocimiento. 
Quienes sirvieron como unidades de análisis fueron docentes y estudiantes de maestría o doctorado relacionados con educación, seleccionados de diferentes universidades. Se tomó una muestra de 18 docentes voluntarios.

Para la recolección de información se utilizaron dos instrumentos los cuales fueron creados por el grupo de investigación, el primero de ellos, fue denominado matriz de categorías que se construyó a partir del fundamento teórico y de discusiones grupales, el cual permitió estructurar el análisis de la información, y el segundo instrumento, consistente en una encuesta semiestructurada de 12 preguntas abiertas la cual indaga sobre los aspectos relacionados con la educación superior a partir de la experiencia personal y del proceso de construcción académica a lo largo de la labor y desempeño profesional elaboradas según las categorías de análisis docente y estudiante.

A partir del planteamiento teórico y metodológico de la investigación, ésta se desarrolló en las siguientes fases:

Fase 1. Una vez recopilada la información pertinente de análisis, se procedió a establecer las diferentes categorías de análisis por medio de discusiones y consultas en los aspectos teóricos y empíricos encontrados, esta categorización se realizó en una matriz denominada "matriz de categorías".

Fase 2. Luego, partiendo de las categorías, se procedió a estructurar la encuesta de pregunta abierta, involucrando preguntas por cada una de las categorías existentes y extraídas de la "matriz de categorías". El instrumento contó con su respectiva validez de contenido, lo que permitió decantar algunas preguntas y diseñar la encuesta definitiva.

Fase 3. Posterior a la aplicación de la encuesta, la información recopilada se llevó a una base o tabla de registro, donde se estructuraron o se conformaron las diferentes categorías ya existentes y las emergentes; éste procedimiento se llevó a cabo por medio de un análisis de citas, códigos, memos, redes, familias y superfamilas y supercódigos. Éste proceso de sistematización y análisis cualitativo de los resultados del proceso de las encuestas, se realizó mediante el software de apoyo en la investigación cualitativa en ciencias sociales Atlas Ti 6.1.

Fase 4. En este parte se analizaron los resultados en tres etapas: la primera, hace referencia al análisis de las 12 respuestas de los encuestados, la segunda, hace referencia al cuadro comparativo de los resultados de las encuestas y la definiciones de las categorías desde el planteamiento del problema, y la tercera etapa se refiere al análisis comparativo de semejanzas y diferencias entre lo reportado por los docentes y lo estructurado desde el marco teórico de la investigación. 


\section{Categorización y análisis de los datos cualitativos}

\begin{tabular}{|c|c|c|c|c|c|}
\hline CATEGORÍA & DEFINICIÓN & SUBCATEGORÍA & DEFINICIÓN & CÓDIGO & PREGUNTAS \\
\hline \multirow[t]{5}{*}{ DOCENTE } & \multirow[t]{5}{*}{$\begin{array}{l}\text { Es un profesio- } \\
\text { nal reflexivo, } \\
\text { es decir, se } \\
\text { concibe más } \\
\text { allá de los } \\
\text { límites conven- } \\
\text { cionales de la } \\
\text { competencia } \\
\text { profesional } \\
\text { (Schön, 1998). }\end{array}$} & CRÍTICO & $\begin{array}{c}\text { Combina la } \\
\text { reflexión y la } \\
\text { práctica acadé- } \\
\text { micas con el fin } \\
\text { de educar a los } \\
\text { estudiantes para } \\
\text { que sean ciuda- } \\
\text { danos reflexivos } \\
\text { y activos. }\end{array}$ & DOC-CRI & $\begin{array}{c}\text { ¿Cuándo } \\
\text { considera que } \\
\text { un docente es } \\
\text { crítico? }\end{array}$ \\
\hline & & $\begin{array}{l}\text { INTELECTUAL } \\
\text { TRANSFORMADOR }\end{array}$ & $\begin{array}{c}\text { Busca la manera } \\
\text { de hacer que la } \\
\text { transformación } \\
\text { de la realidad y } \\
\text { del cambio de } \\
\text { mentalidad de } \\
\text { los educadores } \\
\text { y teóricos, forme } \\
\text { parte de una } \\
\text { sola tarea y, por } \\
\text { supuesto, esto } \\
\text { significa que hay } \\
\text { que implicarse } \\
\text { de lleno en la } \\
\text { lucha política. }\end{array}$ & $\begin{array}{l}\text { DOC-INT- } \\
\text { TRAN }\end{array}$ & $\begin{array}{c}\text { ¿Qué acciones } \\
\text { realiza un } \\
\text { docente crítico? } \\
\text { ¿Cómo lo reco- } \\
\text { noce? }\end{array}$ \\
\hline & & EDUCADOR & $\begin{array}{l}\text { Quien desarrolla } \\
\text { una pedagogía } \\
\text { crítica en la que } \\
\text { se enseñen y } \\
\text { practiquen el } \\
\text { conocimiento, } \\
\text { los hábitos y las } \\
\text { aptitudes de una } \\
\text { ciudadanía críti- } \\
\text { ca, más que de } \\
\text { una mera buena } \\
\text { ciudadanía. }\end{array}$ & DOC-EDU & \\
\hline & & FORMADOR & $\begin{array}{c}\text { Estructura cultu- } \\
\text { ral donde están } \\
\text { gran variedad } \\
\text { de intereses y } \\
\text { practicas en una } \\
\text { lucha constante } \\
\text { y caotica en pos } \\
\text { de la domina- } \\
\text { cion. }\end{array}$ & DOC-FOR & \\
\hline & & ESTUDIANTE & $\begin{array}{l}\text { Profesional } \\
\text { reflexivo que } \\
\text { indaga el } \\
\text { conocimiento } \\
\text { para aprenderlo } \\
\text { y comprenderlo } \\
\text { para luego } \\
\text { ensenarlo. }\end{array}$ & DOC-EST & \\
\hline
\end{tabular}




\begin{tabular}{|c|c|c|c|c|c|}
\hline CATEGORIA & DEFINICION & SUBCATEGORIA & DEFINICION & CODIGO & PREGUNTAS \\
\hline DOCENTE & $\begin{array}{l}\text { Es un profesio- } \\
\text { nal reflexivo, } \\
\text { es decir, se } \\
\text { concibe más } \\
\text { allá de los } \\
\text { límites conven- } \\
\text { cionales de la } \\
\text { competencia } \\
\text { profesional } \\
\text { (Schön, 1998). }\end{array}$ & UNIVERSITARIO & $\begin{array}{l}\text { Es un profesional } \\
\text { crítico, reflexivo } \\
\text { de su propia } \\
\text { práctica, tiene } \\
\text { necesariamente } \\
\text { que investigar } \\
\text { los proceso de } \\
\text { enseñanza- } \\
\text { aprendizaje en } \\
\text { aras de provocar } \\
\text { una reflexión au- } \\
\text { tocrítica dirigida } \\
\text { a su perfecciona- } \\
\text { miento. }\end{array}$ & DOC-UNI & $\begin{array}{c}\text { ¿Cómo } \\
\text { caracteriza un } \\
\text { docente univer- } \\
\text { sitario? ¿Qué } \\
\text { lo diferencia de } \\
\text { un docente en } \\
\text { la educación } \\
\text { secundaria? }\end{array}$ \\
\hline ESTUDIANTE & $\begin{array}{l}\text { Persona capaz } \\
\text { de analizar } \\
\text { problemas y } \\
\text { cuestiones } \\
\text { que tienen } \\
\text { que ver con } \\
\text { los contextos } \\
\text { inmediatos de } \\
\text { la vida y que } \\
\text { debe apropiar- } \\
\text { se críticamente } \\
\text { de formas de } \\
\text { conocimiento } \\
\text { que existen } \\
\text { al margen de } \\
\text { la experiencia } \\
\text { directa de } \\
\text { esa vida, a fin } \\
\text { de ampliar la } \\
\text { facultad de } \\
\text { comprensión y } \\
\text { posibilidad de } \\
\text { si mismos. }\end{array}$ & $\begin{array}{c}\text { ROL } \\
\text { EMPODERAMIENTO }\end{array}$ & $\begin{array}{c}\text { Cuando el estu- } \\
\text { diante producen } \\
\text { significados que } \\
\text { legitiman a for- } \\
\text { mas particulares } \\
\text { de vida. } \\
\text { Personas que } \\
\text { aprender a } \\
\text { correr riesgos, } \\
\text { entender el } \\
\text { pensamiento } \\
\text { diferenciado } \\
\text { del poder como } \\
\text { fuerza produc- } \\
\text { tiva y fuerza } \\
\text { dominadora, ser } \\
\text { capaces de leer } \\
\text { el mundo desde } \\
\text { una diversidad } \\
\text { de perspectivas } \\
\text { y estar dispues- } \\
\text { tos a trascender. }\end{array}$ & $\begin{array}{l}\text { EST-ROL } \\
\text { EST-EMP }\end{array}$ & $\begin{array}{l}\text { ¿Cuál es } \\
\text { perfil ideal que } \\
\text { debe tener un } \\
\text { estudiante en su } \\
\text { asignatura? }\end{array}$ \\
\hline
\end{tabular}

\section{Análisis de los resultados cualitativos}

En este apartado se presenta la información sistematizada y analizada de forma cualitativa, con el fin de determinar la relación entre las teorías en torno a la perspectiva del docente y el estudiante como categorías de análisis de la investigación. En la primera parte de resultados, se analizan las respuestas de los encuestados a partir de las categorías de análisis (docente y estudiante, lo que permitió estructurar a partir de los datos una categorización en tres componentes principales, los cuales se denominan superfamilias, que a su vez, quedaron conformadas por la formación docente. Esta superfamilia, o agrupación de categorías estructuradas a partir de las redes, permiten generar acercamientos teóricos alrededor de la formación del docente universitario.

\section{Formación docente}

En la formación de esta superfamilia quedaron agrupadas las siguientes preguntas: ¿Cómo caracteriza a un docente universitario?, ¿Cuándo considera 
que un docente es crítico?, ¿Qué acciones realiza un docente crítico y cómo lo reconoce?, ¿Cuál es el perfil ideal que debe tener un estudiante de maestría o doctorado en educación?

Frente a la primera pregunta, se evidencia en los informantes que participaron de la encuesta que tienen una dificultad para caracterizar un docente universitario, por cuanto se mueven frente a la dicotomía de lo que en la actualidad es y lo que debería ser característico de dicho docente. En este sentido, el docente universitario se ve como un profesional con un alto nivel de formación que intenta mediar los procesos de aprendizaje de los estudiantes, generando procesos de formación orientados a preparar con altos estándares de calidad a los futuros profesionales, por lo que le corresponde conocer suficientemente la disciplina que enseña. Esto hace del docente universitario una persona que maneja información y unos conocimientos en áreas en las que ha alcanzado idoneidad para llevar a otros a disciplinarse o especializarse en un campo del saber, y por tanto, para desempeñarse en determinado campo socioeconómico.

Ahora, frente a la consideración de cuándo un docente es crítico, los encuestados señalan que son caracterizaciones contextuales de un docente crítico agenciar procesos de formación, no desde verdades absolutas sino cuando como docente es capaz de ver su propio punto de visto como uno, permitiendo valorar las opiniones de los demás, reconociendo que la realidad se compone de múltiples verdades valores, y variadas formas de conocimiento.

Es crítico cuando es capaz de cuestionarse así mismo, siendo observador y analista no sólo de las acciones de sus estudiantes sino de sus propias actuaciones. Además favorece la interdisciplinariedad, esto es, cuando se apoya de los saberes de los demás para hacer más enriquecedor su trabajo docente. De este modo, y en correspondencia con la pregunta ¿Qué acciones realiza un docente crítico y cómo lo reconoce?, se puede entrever una connotación ejemplar del docente, es decir, un docente crítico deviene como un modelo y ejemplo de vida, reflexión y actuación frente al mundo.

Sin embargo, emergen diversas acciones que caracterizan a un docente crítico, teniendo como punto de partida el actuar y devenir profesional propio, es decir, reflexiona permanentemente sobre su actuación, y fundamenta su acción docente en conocer a fondo el campo disciplinar que orienta, investiga, construye, proyecta, y da participación a los estudiantes en estos mismos procesos. Además, indaga sobre la situación social de la comunidad educativa, no desde la simple crítica como cuestionamiento sino que desarrolla procesos pedagógicos que contextualizan y promueven una lectura crítica y reflexiva de la realidad social, política, económica, ética y moral en su entorno. Dicha lectura crítica permite materializarse en sus mismas prácticas educativas, es decir, permite que sus estudiantes cuestionen su quehacer docente y facilita que éstos se sientan incluidos dentro de la misma. 
La docencia universitaria con miras a la comprensión de la realidad: una mirada desde el enfoque sociocrítico. Artículo original producto de la investigación.

En el proceso de encuesta, se buscó indagar en los participantes la comprensión sobre el perfil ideal que debe tener un estudiante en un nivel posgradual de educación, de modo que se formuló la pregunta ¿Cuál es el perfil ideal que debe tener un estudiante de maestría o doctorado en educación? Como elementos básicos para dicho perfil emergen el sentido y la importancia, por un lado, de haber adquirido en su formación pre gradual una comprensión conceptual clara frente a los temas pertinentes que desde la educación debe abordarse, y por otro lado, tener vocación para la enseñanza y la reflexión educativa, y una disposición sistemática para la actualización disciplinaria de su quehacer profesional.

Ahora, en los aspectos relacionados con la segunda parte de los resultados, se compara a modo de resumen la conceptualización de los informantes con la información encontrada en el marco de referencia que sustenta la investigación, la que queda conformada de la siguiente forma, tabla 1.

Dentro de estos resultados, se tuvieron en cuenta los aspectos teóricos específicos de las categorías docente y estudiante según lo conceptualizado por Giroux y las respuestas a estas mismas, evidenciadas en las encuestas a los docentes y estudiantes de educación superior con maestría o doctorado en educación. Como resultados de esta información se realizó un análisis de la investigación y se hizo el comparativo entre los resultados de las respuestas resumidas de los informantes y la conceptualización sociocrítica de las categorías.

En la primera de las categorías, que hace referencia al docente, Tabla 1, las diferencias emergen en tanto desde los informantes se toma como un profesional con un alto nivel de formación y desde el enfoque sociocrítico de Giroux, como alguien que ejerce una práctica intelectual y pedagógica con sentido social. Ambos coinciden en que el docente presenta influencia en el estudiante.

Tabla 1. Comparativo de concepto de docente

\begin{tabular}{|c|c|}
\hline Informantes & Giroux \\
\hline $\begin{array}{l}\text { Se ve como un profesional con un alto nivel de formación } \\
\text { que intenta mediar los procesos de aprendizaje de los es- } \\
\text { tudiantes, generando procesos de formación orientados } \\
\text { a preparar con altos estándares de calidad a los futuros } \\
\text { profesionales, por lo que le corresponde conocer suficien- } \\
\text { temente la disciplina que enseña. Esto hace del docente } \\
\text { universitario una persona que maneja información y unos } \\
\text { conocimientos en áreas que además ha alcanzado ido- } \\
\text { neidad para llevar a otros a disciplinarse o especializarse } \\
\text { en un campo del saber, y por tanto, para desempeñarse } \\
\text { en determinado campo socioeconómico. }\end{array}$ & $\begin{array}{l}\text { "La expresión intelectual transformador se refiere a aquel } \\
\text { que ejerce formas de práctica intelectual y pedagógica } \\
\text { que intentan insertar la enseñanza y el aprendizaje } \\
\text { directamente en la esfera política, argumentando que } \\
\text { la enseñanza representa tanto una lucha en pos del } \\
\text { significado como una pugna en torno a las relaciones } \\
\text { de poder. También me refiero a alguien cuyas prácticas } \\
\text { intelectuales se hallan necesariamente fundamentadas } \\
\text { en formas de discurso moral y ético que manifiestan una } \\
\text { preocupación preferencial por el sufrimiento y las luchas } \\
\text { de los desposeídos y los oprimidos" (Giroux, 1993, p 26). } \\
\text { "El concepto del docente como intelectual trae aparejado } \\
\text { el imperativo de juzgar, criticar y rechazar los enfoques } \\
\text { de la autoridad que refuerzan una división técnica y social } \\
\text { del trabajo que silencia y priva de poder tanto a docentes } \\
\text { como a alumnos" (Giroux, 2003, p. 154). }\end{array}$ \\
\hline
\end{tabular}


Respecto a la relación que se establece en el concepto de estudiante, tabla 2, se observa que en la primera se trabaja como el que recibe una información y la interpreta conceptualmente y el segundo lo ve como aquel que asume riesgos a partir de un pensamiento abierto. En sus similitudes se puede decir que los dos lo ven como aquel que tiene una disposición de aprender.

Tabla 2. Comparativo de concepto de estudiante

\begin{tabular}{|c|c|}
\hline Informantes & Giroux \\
\hline $\begin{array}{c}\text { El perfil que emerge a partir de los informantes parte de } \\
\text { hallar el sentido y la importancia, por un lado, de haber } \\
\text { adquirido en su formación pre gradual una comprensión } \\
\text { conceptual clara frente a los temas pertinentes que desde } \\
\text { la educación debe abordarse, y por otro lado, tener vo- } \\
\begin{array}{c}\text { cación para la enseñanza y la reflexión educativa, y una } \\
\text { disposición sistemática para la actualización disciplinaria } \\
\text { de su quehacer profesional. }\end{array}\end{array}$ & $\begin{array}{c}\text { "los alumnos y otras personas tienen que aprender a } \\
\text { correr riesgos, entender el pensamiento diferenciado } \\
\text { ser capaces de leer el mundo desde una diversidad de } \\
\text { perspectivas y estar dispuestos a trascender" (Giroux, } \\
\text { 2003, p. 239). }\end{array}$ \\
\hline
\end{tabular}

\section{Discusión}

En este apartado se contrasta desde el fundamento teórico hasta lo encontrado partiendo del hecho que no es posible transformar los procesos educativos y las políticas educativas, ni mejorar la calidad educativa, si no se parte de transformar y mejorar los procesos de formación de quienes tienen en sus manos o tendrán la función de educar a los futuros docentes. Es decir, el punto clave de dicha transformación está en mejorar los procesos de formación de formadores, por lo cual una reflexión sobre los lineamientos que orientan los procesos de formación superior en la actualidad, desde una perspectiva sociocrítica, puede orientar la formación docente.

\section{Formación sociocrítica del docente universitario}

Dado que a partir de los resultados de la encuesta realizada se evidencia un rol y un perfil del docente universitario como ejercicio profesional que tiene como objeto de sus acciones la consolidación de estándares de los futuros profesionales, a partir de una disciplina en particular, por lo que centra su atención en manejar suficiente información para llevar a otros a especializarse en la misma, pero sobre todo, para consolidar un perfil de desempeño competente en determinado campo socioeconómico y teniendo en cuenta que las políticas neoliberales y globalizantes han permeado los centros educativos y los han convertido en burbujas de conocimientos teóricos, adquiere significación preguntar ¿Quién forma a los docentes? Las dinámicas actuales, caracterizadas, por ejemplo, por el integracionismo cultural, el aislacionismo social, la tecnologización mediática; reclaman una reflexión crítica y humanizadora de la formación de los futuros docentes. 
Por esto, pensar al docente como un intelectual revolucionario, transformador, como lo propone Giroux, es advertir que sus prácticas deben situarse en contexto con los hechos sociales y en respuesta a las problemáticas que le rodean. Es advertir que los procesos educativos que desarrolla deben sugerir una formación crítica que dé significado a la vida de sus estudiantes. La educación no puede ser un aspecto periférico de lo humano.

La universidad, como se ha mencionado respecto del estudiante, también debe asumir un empoderamiento, una toma de conciencia de su rol social y cultural en el progreso de una nación. Desde luego es un avance la estructuración de la proyección social de los centros universitarios, pero esto debe constituirse en un punto inicial de construcción social. La universidad debe comprenderse a sí misma como un sujeto social.

Los docentes, como sujetos de trasformación social, requieren de una formación que favorezca una visión de la escuela y de la educación como referentes críticos de construcción política y social, y que promuevan la formación de estudiantes como ciudadanía crítica. Esto trae consigo el desarrollo de programas curriculares interdisciplinarios y contextualizados. Para Giroux (2006), el docente debe ser ante todo un intelectual trasformador, conocedor de lo que enseña y promotor de la formación escolar de sus estudiantes como investigadores reflexivos capaces de trabajar en favor de la comunidad donde se hallan inmersos.

De ahí que, no es un imaginario que la formación docente, más que la labor docente, quizá en toda Latinoamérica, presenta una situación de falta de significatividad, de fragmentación y deficiencia en la formulación de los objetivos, en la gestión de las políticas de gobierno y en la forma en que se desarrollan los procesos tendientes a la formación de los docentes, y a la sostenibilidad de los mismos. En términos profesionales, la labor docente, por ejemplo, ha perdido un carácter significativo, esencial y fundamental en el desarrollo de la sociedad.

El enfoque sociocrítico pone de manifiesto la importancia del papel desarrollado por el docente como modelo de resistencia y empoderamiento, como referente de valores y de transformación a partir de la coherencia entre la forma de educar y la forma de vivir propia. Formar un pensamiento sociocrítico exige un compromiso del docente dentro y fuera del aula. En efecto, la noción tradicional del docente como trasmisor de información debe dar paso a una concepción cívica de éste, como precursor de una formación de sujetos con liderazgo cívico, con compromiso de transformación. Para Giroux, el profesor como intelectual transformador, induce a la fracturación de esquemas de opresión a favor de una visión política de la historia, en el surgir de una conciencia cívica en los estudiantes (2006).

Así, el estudiante como sujeto autónomo, no puede comprenderse como un producto de una educación formalizada ideológicamente, como un agente pasivo de la sociedad, como un objetivo de políticas estatales, sino, por el 
contrario, debe ir incubando serios cuestionamientos sobre el tipo de sociedad de la que desea hacer parte y la manera como ésta le hace partícipe. No se pueden seguir forjando procesos educativos tendientes a la consecución de estándares sino a la humanización y dignificación de la sociedad.

En efecto, se evidencia una escisión entre la formación y la práctica profesional del docente. Esto demanda la necesidad de que el desarrollo curricular aborde este distanciamiento entre teoría y práctica, entre lo que se aprende en el claustro universitario y la forma como se abordan los problemas y las situaciones en el ejercicio laboral.

El enfoque socio-crítico orienta la comprensión de la formación del ser humano como un sujeto dinámico y gestor de mejores relaciones sociales y mejor calidad de vida para todos, como promotor de una lucha frente a los desequilibrios institucionalizados de la cultura de dominio imperante. La pedagogía crítica entrevé la educación como un proceso de transformación de la realidad, de análisis de las situaciones de las escuelas, de la comunidad, de la nación, de participación en la construcción de la sociedad, su cultura, su vida política, a partir de la crítica dialógica y reflexiva. Al respecto, Apple establece la necesidad de generar un desadiestramiento del docente, es decir, un análisis crítico de su acción educativa para que no consista en la reproducción de conocimientos mediante modelos pedagógicos e ideológicos normatizados, que finalmente confluyen en la consolidación de una educación de control técnico-administrativo o de disposición conductual de los estudiantes (Apple, 2000).

La formación docente desde esta perspectiva es pues una acción social transformadora, dirigida a dar sentido a las prácticas escolares, al desarrollo permanente e integral del hombre y a la gestión de un proyecto de nación democrático y justo. Habermas, otro de los grandes precursores de la teoría crítica, propuso la Ciencia Social Crítica como un proyecto emancipador del ser humano, de su integridad, de sus facultades, de su interacción social; mediante la relación dialéctica entre sujeto, sociedad y mundo, para lograr la concientización de la persona, esto es, la autocomprensión e integración de su devenir social, político, histórico e ideológico. Esta ciencia social crítica demanda la creación de un saber racional práctico, autorreflexivo y autónomo, capaz de traducirse en acciones sociales de transformación (Habermas, 1984).

\section{Conclusiones}

La docencia es un proceso de humanización, un proceso de socialización de la persona. De ahí que, la pedagogía crítica ofrece a la formación docente un carácter emancipador, liberador, que lleva a comprender y establecer 
respuestas a las problemáticas relacionadas no sólo con la práctica pedagógica sino también con el universo de la comunidad escolar en las que ésta se desarrolla. Ofrece la reflexión crítica y la toma de conciencia frente al mundo, para formar un sujeto orientado a la transformación social y a la reivindicación de la justicia social.

Desde la pedagogía crítica se concibe al docente como un sujeto que media la formación integral, la humanización y el progreso colectivo de la sociedad. Lo lleva además a apropiarse socialmente de los problemas de su entorno, a atender las necesidades del aprendizaje, a la comprensión e interpretación de la realidad como constructo social y a asumir un compromiso social y político mediante el ejercicio de su profesión. Lleva asimismo a favorecer la reflexión histórica de su quehacer educativo.

Además, esta perspectiva de la pedagogía crítica, suscita una epistemología que invita al docente a hacerse crítico reflexivo, a apropiarse de las contradicciones que se desarrollan en la cotidianidad, a promover el desarrollo de la autoconciencia, a cultivar un actuar emancipador, a pensarse como hacedor de la historia, como agente de la democracia, en la praxis de una ciudadanía crítica y en búsqueda de una mejor sociedad.

\section{Referencias bibliográficas}

Apple, M. (1986). Ideología y currículo. Madrid: Ediciones AKAL.

Apple, M. (2000). Teoría Crítica y Educación. Buenos Aires: Niño y Dávila Editores.

Freire, P. (1985). Pedagogía del oprimido. México: Siglo XXI.

Freire, P. (1997). Política y educación. México: Siglo XXI.

Giroux, H. (1997). Los profesores como intelectuales: Hacia una pedagogía crítica del aprendizaje. España: Paidós.

Giroux, H. (2003). Pedagogía y política de la esperanza. Traducción de Horacio Pons. Buenos Aires: Amorrortu.

Giroux, H. (2006). La escuela y la lucha por la ciudadanía. Traducción de Martín Mur Ubasart. México: Siglo XXI.

Habermas, J. (1984). Ciencia y técnica como ideología. Madrid: Tecnos.

McLaren, P. (1997). Pedagogía crítica y cultura depredadora. Barcelona: Paidós. 


\section{Revista de}

investigaciones UNAD

Volumen 11. Número 2. Julio 2012

McLaren, P. (2001). El Che Guevara, Paulo Freire y la pedagogía de la revolución. Buenos Aires: Siglo XXI.

McLaren, P. (2003). La vida en las escuelas. Una introducción a la pedagogía crítica en los fundamentos de la educación. México: Siglo XXI.

Strauss, A. \& Corbin, J. (1998). Bases de la investigación cualitativa, técnicas y procedimientos para desarrollar la teoría fundamentada. Colombia: Universidad de Antioquia. 